\title{
ESTABILIZACIÓN POR VERMICOMPOSTEO DE LODOS RESIDUALES APLICADOS EN LA PRODUCTIVIDAD DE ALBAHACA (Ocimum basilicum L.)
}

\author{
Stabilization of sewage sludge by vermicomposting applied in the productivity of basil \\ Diana Yatzil REYES ARAUJO ${ }^{1}$, Martha Elena MORA HERRERA ${ }^{1 *}$, \\ Jorge LUGO $^{2}$ y Pedro DEL ÁGUILA ${ }^{2}$
}

\author{
${ }^{1}$ Centro Universitario Tenancingo, Universidad Autónoma del Estado de México, km 1.5 carretera Tenancingo- \\ Villa Guerrero, 52400 Tenancingo, Estado de México, México \\ ${ }^{2}$ Facultad de Ciencias, Universidad Autónoma del Estado de México, Instituto Literario 100, Col. Centro, 50000 \\ Toluca, Estado de México, México \\ *Autora para correspondencia: marthaelenam@gmail.com
}

(Recibido: febrero 2019; aceptado: agosto 2019)

Palabras clave: fertilización inorgánica, Eisenia foetida, vermicompostaje

\section{RESUMEN}

Los lodos residuales, subproducto del tratamiento de las aguas residuales, acumulan un alto contenido de materia orgánica, metales pesados y otros compuestos tóxicos. Debido a su acelerada generación, es necesario establecer un manejo adecuado para su estabilización y disposición. El vermicomposteo es una alternativa de manejo que estabiliza los lodos residuales y permite su adición al suelo, mejorando las propiedades estructurales e incrementando su fertilidad. El presente estudio tuvo como objetivo evaluar el efecto de vermicomposta de lodo residual y estiércol equino en la productividad y actividad antioxidante de plantas de albahaca (Ocimum basilicum L.). Se elaboró vermicomposta en proporción 30:70 de lodo residual y estiércol equino, y se evaluó el efecto en albahaca con diferentes dosis de vermicomposta $(0,20,40$ y $60 \mathrm{t} / \mathrm{ha})$. El uso de vermicomposta incrementó significativamente la tasa de crecimiento, sin ocasionar efectos en la actividad antioxidante y el contenido de compuestos fenólicos en las plantas de albahaca.

Key words: inorganic fertilization, Eisenia foetida, vermicomposting

\begin{abstract}
Sewage sludge, a byproduct of wastewater treatment, accumulates a high content of organic matter, heavy metals and other toxic compounds. Due to its accelerated generation, adequate management is necessary for its stabilization and disposal. Vermicomposting is a management alternative that stabilizes residual sludge and allows its addition to the soil, improving structural properties and increasing its fertility. The objective of this study was to evaluate the effect of vermicompost of sewage sludge and equine manure on the productivity and antioxidant activity of basil plants (Ocimum basilicum L.). Vermicompost was developed in a 30:70 proportion of sewage sludge and equine manure, and the effect on basil was evaluated under different doses of vermicompost $(20,40$ and $60 \mathrm{t} / \mathrm{ha})$. The use of vermicompost significantly increased the growth rate, without causing effects on the antioxidant activity and the content of phenolic compounds in basil plants.
\end{abstract}




\section{INTRODUCCIÓN}

El acelerado aumento demográfico, la diversidad y complejidad de los procesos industriales y los requerimientos agroindustriales, han incrementado considerablemente el uso del agua, que en su reintegración a la naturaleza frecuentemente contiene contaminantes que pueden alterar las condiciones para su reutilización (BID 2013, Holguín-Calderón et al. 2014, Lim et al. 2016).

Las plantas tratadoras de aguas residuales se han establecido con el objetivo de remover los contaminantes del agua y reutilizarla sin causar daños al ambiente. Durante este proceso se genera como subproducto lodo residual, que acumula materia orgánica, metales pesados y otros compuestos tóxicos que no pueden ser removidos durante el tratamiento (BID 2013, Holguín-Calderón et al. 2014). Las concentraciones elevadas de metales en el ambiente causan fitotoxicidad, que se traduce en estrés oxidativo celular que produce especies reactivas de oxígeno (ERO), lo que ocasiona por un lado daño oxidativo e inclusive muerte celular y por otro cambios en el sistema de defensa antioxidativa de las plantas (Charles 2012, Cuypers et al. 2013, Carbonell et al. 2016).

Una alternativa de manejo de los lodos residuales que reduzca el impacto ambiental es la estabilización mediante vermicomposteo y su incorporación a los suelos. El uso de vermicomposta cambia las propiedades físicas, químicas y biológicas del suelo; aumenta la biomasa microbiana y mejora el crecimiento y desarrollo de las plantas (Arancon y Edwards 2011, Vicencio-de la Rosa et al. 2011, Holguín-Calderón et al. 2014).

La disposición de los lodos residuales mediante la aplicación directa al suelo o después de la estabilización, ha contribuido a la recuperación de materia orgánica (Alloway 2013); también se han demostrado los beneficios de usar lodos residuales como abono orgánico en el aumento del rendimiento y productividad de diversos cultivos (INIFAP 2004, Alloway 2013, Rodríguez et al. 2018).

Se ha encontrado que la aplicación de lodos residuales al suelo como abono aumenta el rendimiento de alfalfa, además de incrementar la producción de 17 a $31 \%$, incluso mayor a la obtenida por fertilizantes inorgánicos (INIFAP 2004). Utria-Borges et al. (2008) encontraron que en cultivos de tomate fertilizados con lodos residuales se incrementa el área foliar, altura de la planta, diámetro del tallo y peso seco, y en rábano, la incorporación de lodos residuales aumenta el rendimiento (Rodríguez et al. 2018).
Por otro lado, se ha demostrado que el uso de vermicomposta tiene efectos beneficiosos para los cultivos en general; por ejemplo, en el cultivo de albahaca se presenta un incremento en la biomasa, número y área de hojas y el contenido de clorofila en comparación con el uso de fertilizantes químicos (Cabanillas et al. 2013). El objetivo de este trabajo fue estabilizar lodos residuales a través de vermicomposteo y evaluar su productividad y actividad antioxidante en plantas de albahaca (Ocimum basilicum L.).

\section{MATERIALES Y MÉTODOS}

El estudio se llevó a cabo de agosto a noviembre de 2017 en un invernadero no tecnificado del Centro Universitario Tenancingo de la Universidad Autónoma del Estado de México, ubicado en el km 1.5 de la carretera Tenancingo-Villa Guerrero, Estado de México.

Se monitoreó la temperatura media, humedad relativa e intensidad lumínica utilizando un termómetro digital con rangos de $-35-120{ }^{\circ} \mathrm{C}$ marca Data logger ANA modelo IP67 con sensores de temperatura y humedad, programado para tomar lectura cada $2 \mathrm{~h}$ (Cuadro I).

\begin{tabular}{lccc} 
CUADRO I. & CONDICIONES CLIMÁTICAS DE LA LO- \\
& CALIDAD DE SANTA ANA, TENANCINGO \\
& DEGOLLADO DURANTE LOS MESES DE \\
& ESTUDIO PARA LA PRODUCTIVIDAD \\
& $\begin{array}{l}\text { DEL CULTIVO DE ALBAHACA (Ocimum } \\
\text { basilicum L.) }\end{array}$ \\
& & & \\
\hline Mes & Temperatura & Humedad & Intensidad \\
& media $\left({ }^{\circ} \mathrm{C}\right)$ & relativa (\%) & lumínica (Lux) \\
\hline Ago-17 & 20.25 & 75.06 & 1640.92 \\
Sep-17 & 18.39 & 80.98 & 825.32 \\
Oct-17 & 16.69 & 78.24 & 649.36 \\
Nov-17 & 13.61 & 73.80 & 560.66 \\
\hline
\end{tabular}

La preparación de la vermicomposta se realizó en dos fases: el precomposteo y el vermicomposteo. Se elaboró una mezcla inicial de lodo residual proporcionado por la planta tratadora de aguas residuales municipales Toluca Norte, Operadora de Ecosistemas -el cual se pasó por un tamiz de $2 \mathrm{~mm}-\mathrm{y}$ estiércol de caballo (Garg et al. 2005) obtenido del Hípico de la Facultad de Veterinaria y Zootecnia de la UAEM, en proporción 30:70 (v/v) sugerida por Lugo et al. (2017). Estos autores mencionan que la relación de $30 \%$ delodo residual y $70 \%$ de estiércol 
equino presenta un $\mathrm{pH}$ neutro y gran aporte de $\mathrm{C} \mathrm{y}$ $\mathrm{N}$, recomendable como acondicionador de suelo con condiciones adecuadas para el desarrollo de Eisenia foetida.

\section{Precomposteo}

El precomposteo consistió en la fermentación de la mezcla inicial, colocada en contenedores plásticos de $20 \mathrm{~L}$, con una cubierta negra plástica con perforaciones. Las condiciones de oscuridad, humedad y aireación se mantuvieron controladas. La temperatura fue monitoreada una vez por semana con un termómetro estándar de mercurio. El pH se evaluó cada tercer día (por triplicado) en el lixiviado de la mezcla con un pHmetro THERMO marca Orion Star A215, medidor multiparamétrico de $\mathrm{pH} / \mathrm{mV} / \mathrm{RmV}$ o conductividad/TDS/salinidad/resistividad con temperatura. El proceso de precomposteo se detuvo a los 20 días, una vez que se alcanzaron los parámetros de $\mathrm{pH}$ y temperatura adecuados para incorporar las lombrices.

\section{Vermicomposteo}

$\mathrm{Al}$ alcanzar un $\mathrm{pH}$ cercano a la neutralidad durante el proceso de precomposteo, de acuerdo con Romero et al. (2018), se adicionaron a la mezcla 25 lombrices jóvenes en edad reproductiva con una longitud de 3 a $5 \mathrm{~cm}$ en un recipiente con un volumen de $10 \mathrm{~L}$.

Para determinar la estabilidad de la vermicomposta se realizó la evaluación de parámetros como $\mathrm{pH}$, conductividad eléctrica (CE) y materia orgánica (MO) cada 15 días a partir de la adición de las lombrices y hasta su maduración.

Para conocer el efecto de la vermicomposta sobre la productividad de Ocimum basilicum L. se empleó un diseño en bloques, con cinco tratamientos y seis repeticiones. Cada repetición constó de cinco plantas, con un total de 30 plantas por tratamiento. Los tratamientos evaluados fueron: $\mathrm{C}$ (control absoluto: peat moss + agrolita 2:1), $\mathrm{CT}_{0}$ (control relativo: suelo agrícola), $\mathrm{T}_{20}$ (suelo + vermicomposta a $20 \mathrm{t} / \mathrm{ha}$ ), $\mathrm{T}_{40}$ (suelo + vermicomposta a $40 \mathrm{t} / \mathrm{ha}$ ) y $\mathrm{T}_{60}$ (suelo + vermicomposta a $60 \mathrm{t} / \mathrm{ha}$ ).

\section{Evaluación de la productividad}

Para evaluar el efecto de la vermicomposta en la productividad de la albahaca, se utilizaron semillas con categoría declarada de la variedad fina verde, con $90 \%$ de germinación y $99 \%$ de pureza, obtenidas de la distribuidora Rancho Los Molinos, con número de lote 1094. Las semillas fueron germinadas en sustrato de agrolita y peat moss (2:1). El trasplante se realizó en macetas con agrolita y suelo agrícola (cultivado por seis años sin descanso, con coordenadas $19^{\circ} 10^{\prime}$ 00 " $\mathrm{N}$ y $99^{\circ} 65^{\prime} 80^{\prime \prime} \mathrm{O}$, a $2880 \mathrm{msnm}$ ) y se adicionó la vermicomposta en dosis de $0,20,40$ o 60 t/ha. Las macetas se ubicaron en camas a una altura de $90 \mathrm{~cm}$; el área total del experimento $\left(2.5^{\prime} 5^{\prime} 4 \mathrm{~m}\right)$ se cubrió con tela de organza antiáfidos. Se evaluó la longitud $(\mathrm{cm})$ y peso fresco $(\mathrm{mg})$ al inicio y antes de la floración (60 días). Los datos se analizaron de acuerdo con los índices de crecimiento empleados en fisiología vegetal y de cultivos propuestos por Hunt (1982).

\section{Cuantificación del contenido de compuestos fe- nólicos totales}

La cuantificación de los compuestos fenólicos totales se realizó mediante el método de Folin-Ciocalteu descrito por Waterman y Mole (1994), a una longitud de onda de $760 \mathrm{~nm}$ utilizando un espectrofotómetro marca Thermo Scientific modelo Genesys 10S UV-Vis equipado con una lámpara de xenón de alta intensidad y una geometría óptica de doble haz.

\section{Cuantificación de la capacidad antioxidante}

La cuantificación de la capacidad antioxidante se realizó mediante el método DPPH (1,1-difenil2-picril-hidracilo) descrito por Abe et al. (1998) a una longitud de onda de $517 \mathrm{~nm}$.

Con los datos se realizó un análisis de varianza (ANOVA) y una comparación de medias (Tukey) mediante el programa estadístico InfoStat-Statistica.

\section{RESULTADOS Y DISCUSIÓN}

\section{Caracterización de sustratos}

Se realizó un análisis tanto de la composición de los sustratos empleados para elaborar la vermicomposta como del suelo agrícola utilizado para este experimento (Cuadro II).

Estos indicadores intrínsecos del suelo, como $\mathrm{pH}$, contenido de arcilla (textura), MO y CE son componentes del suelo que influyen en el suministro, biodisponibilidad, persistencia, movimiento y efecto de nutrientes o de algunos contaminantes, y son también características esenciales para alcanzar el potencial de crecimiento de las plantas (Bloem et al. 2006, Havlin y Moebius-Clune 2012).

El suelo utilizado en esta investigación está clasificado como fluvisol (INEGI 2007); presenta una textura franco arenosa, con un porcentaje de arena de 78.2, limo 12.9 y arcilla 8.9 , que de acuerdo con la clasificación del United States Department of Agriculture (USDA) corresponde a un suelo de textura 
CUADRO II. DETERMINACIÓN DEL CONTENIDO DE MATERIA ORGÁNICA(MO), pH, CONDUCTIVIDAD ELÉCTRICA (CE) Y TEXTURA DEL SUELO AGRÍCOLA, DEL LODO RESIDUAL Y DEL ESTIÉRCOL EQUINO, ANTES DE LA ELABORACIÓN DE LA VERMICOMPOSTA

\begin{tabular}{lccccc}
\hline Sustratos & $\mathrm{MO}(\%)^{*}$ & $\mathrm{pH}^{*}$ & $\mathrm{CE}(\mathrm{dS} / \mathrm{m})^{*}$ & Textura \\
\hline Suelo & $2.7 \pm 0.2$ & $5.6 \pm 0.07$ & $1.18 \times 10-3 \pm 0.5$ & Franco arenoso \\
Lodo residual & $30.4 \pm 0.4$ & $7.1 \pm 0.4$ & 3 & \pm 0.2 & - \\
Estiércol equino & $8.62 \pm 0.4$ & $7.4 \pm 0.6$ & $7.00 \pm 0.0$ & - \\
\hline
\end{tabular}

*Promedio \pm error estándar

gruesa, pobre en materia orgánica y nitrógeno, contenidos medios de fósforo y potasio, $\mathrm{pH}$ ligeramente alcalino que aumenta con la profundidad y apto para el cultivo (IUSS 2007). El suelo presentó un porcentaje de 2.7 de $\mathrm{MO}$, que de acuerdo con la clasificación de la NOM-021-RECNAT-2000 (SEMARNAT 2002) es un valor muy bajo. El estiércol equino también tuvo un porcentaje bajo (8.62) de MO; en contraste, el lodo residual, presentó un porcentaje de 30.4.

$\mathrm{El} \mathrm{pH}$ inicial del suelo fue de 5.6, que de acuerdo con la NOM-021-RECNAT-2000 (SEMARNAT 2002), es un suelo moderadamente ácido. Por su parte, el estiércol equino y el lodo residual presentan valores neutros de $\mathrm{pH} 7.1$ y 7.4, respectivamente.

En cuanto a la $\mathrm{CE}$, el suelo presentó valores de $0.0018 \mathrm{dS} / \mathrm{m}$, que están clasificados como "muy ligeramente salinos" de acuerdo con la NOM021-RECNAT-2000 (SEMARNAT 2002), y son aptos para el crecimiento de cultivos en general. En el caso del lodo residual, una $\mathrm{CE}$ de $3 \mathrm{dS} / \mathrm{m}$ le da cualidades "moderadamente salinas", y el estiércol equino, con una CE de $7 \mathrm{dS} / \mathrm{m}$, se clasifica como "fuertemente salino". Estos resultados obtenidos en los sustratos permitieron iniciar el proceso de precomposteo.

\section{Precomposteo}

Durante el proceso de precomposteo, el $\mathrm{pH}$ y la temperatura disminuyeron en los primeros 7-8 días. La estabilización se inició a partir del día 11 (Fig. 1) hasta alcanzar un $\mathrm{pH}$ de 7.6 y una temperatura de $14{ }^{\circ} \mathrm{C}$ en el día 20.

El precomposteo es el periodo previo a la adición de lombrices. Este proceso varía de acuerdo con el origen y propiedades de los sustratos utilizados, por lo que su estabilización depende tanto de las propiedades bioquímicas de los sustratos como de factores externos (temperatura, humedad y $\mathrm{pH}$ ) (Acosta-Durán et al. 2013). Por estas razones no hay un consenso de cuál es la duración precisa, la temperatura y el $\mathrm{pH}$ óptimo del precomposteo. Por ejemplo, David-Santoya et al. (2018) mencionan que
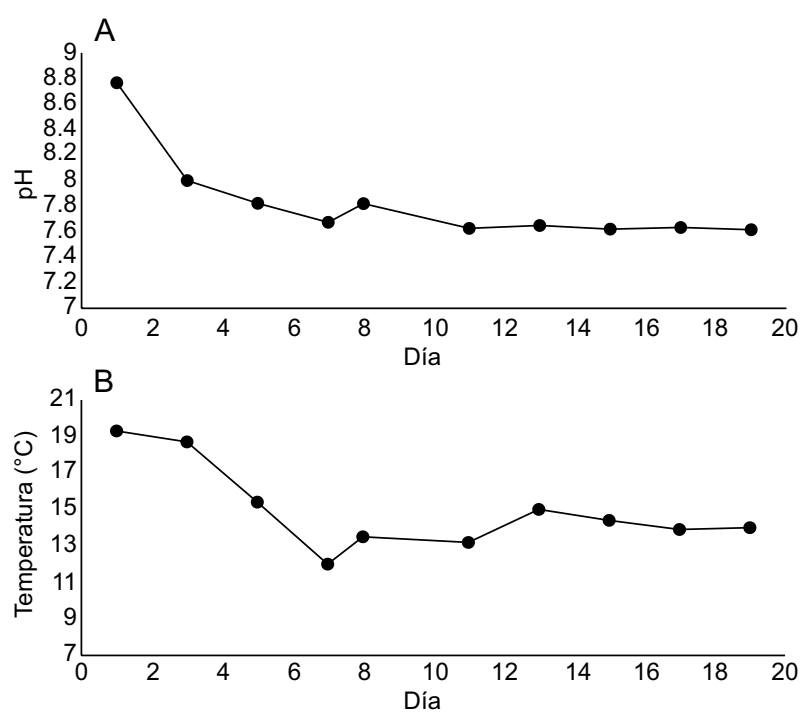

Fig. 1. Monitoreo de (a) pH y (b) temperatura en el proceso de precomposteo del lodo residual y estiércol equino

para vermicomposta elaborada a base de estiércol de ovino, residuos vegetales, bocashí, hojas y tallos de G. sepium, y cascarilla de cacao en diferentes combinaciones, se requiere un periodo de ocho semanas. Por su parte, Vázquez y Loli (2018) afirman que para vermicompostas elaboradas con restos de podas, cortes de césped y frutas, mezcladas con el estiércol de ganado vacuno, se requiere un precomposteo de 60 días (10 semanas).

Para establecer el tiempo necesario de precompostaje, Acosta-Durán et al. (2013) evaluaron diferentes tiempos de precomposteo de estiércol maduro de borrego y residuos orgánicos domésticos frescos. Establecieron que la duración del precomposteo está limitada por el origen de los sustratos utilizados y el objetivo que se busque sobre el desarrollo de la lombriz. En general, el precomposteo de cero a dos semanas favorece la reproducción y el de tres a siete semanas favorece el crecimiento individual de las lombrices. 
De acuerdo con lo anterior y los resultados obtenidos en esta investigación, el precomposteo de la vermicomoposta de lodo residual y estiércol equino, cuya extensión es de 20 días, es un proceso de corta duración que disminuye el gasto de insumos; por lo tanto, abate el costo final de la vermicomposta y acelera la generación de un producto estable y adecuado para su uso.

Respecto a la temperatura, se considera un factor determinante para la adición y sobrevivencia de Eisenia foetida. Los $14{ }^{\circ} \mathrm{C}$ alcanzados durante el precomposteo fueron adecuados para agregar las lombrices, ya que, si bien son relativamente tolerantes a diversas condiciones ambientales, se desarrollan mejor entre 15 y $25^{\circ} \mathrm{C}$. Temperaturas inferiores a 10 ${ }^{\circ} \mathrm{C}$ provocan una alimentación reducida o escasa, $\mathrm{y}$ por debajo de los $4^{\circ} \mathrm{C}$ la producción de capullo y el desarrollo de lombrices jóvenes cesa por completo (Singh et al. 2016).

Como se señaló anteriormente, el pH también es un factor limitante durante el proceso de precomposteo y en general durante la elaboración de la vermicomposta. De acuerdo con ICAMEX (2008), un precomposteo con valores de 5 a 7 son aceptables; en contraste, las especificaciones de la NMX-FF-109SCFI-2008 sugieren valores de $\mathrm{pH}$ en un rango de 5.5 a 8.5 (SAGARPA 2008).

A pesar de los diferentes valores óptimos de duración del proceso, el $\mathrm{pH}$ y la temperatura del precomposteo encontrados en la literatura, los valores obtenidos con lodo residual y estiércol equino se mantuvieron dentro de los parámetros establecidos en las especificaciones físicas y químicas de la NMX-FF-109-SCFI-2008 (SAGARPA 2008). Una vez estabilizado el precomposteo, se integraron las lombrices para iniciar el vermicomposteo.

\section{Vermicomposteo}

El proceso del vermicomposteo concluyó a los 60 días de la adición de lombrices, cuando tanto el porcentaje de materia orgánica como la conductividad eléctrica y el pH se estabilizaron (Fig. 2).

El pH inició en 7.4 y finalizó en 7.06 (Fig. 2a). Este indicador influye en la disponibilidad de nutrientes, ya que la acidez o alcalinidad del sustrato promoverán la disponibilidad de éstos (solubles o insolubles) para su absorción (Havlin y Moebius-Clune 2012). La determinación del rango adecuado de $\mathrm{pH}$ depende de los requerimientos de cada cultivo, ya que si es muy bajo pueden presentarse deficiencias de N, K, Ca y Mg; por ejemplo, un $\mathrm{pH}$ menor a 5 promueve la disponibilidad de óxidos metálicos que pudieran resultar fitotóxicos; en cambio, un $\mathrm{pH}$ muy alto disminuye la solubilidad de $\mathrm{Fe}, \mathrm{P}, \mathrm{Mn}, \mathrm{Cu}$ y $\mathrm{Zn}$. En general, un $\mathrm{pH}$ neutro permite la adsorción de macro y micronutrientes (Havlin y Moebius-Clune 2012).

De acuerdo con diversos autores, el $\mathrm{pH}$ obtenido en vermicompostas de diferentes sustratos presenta

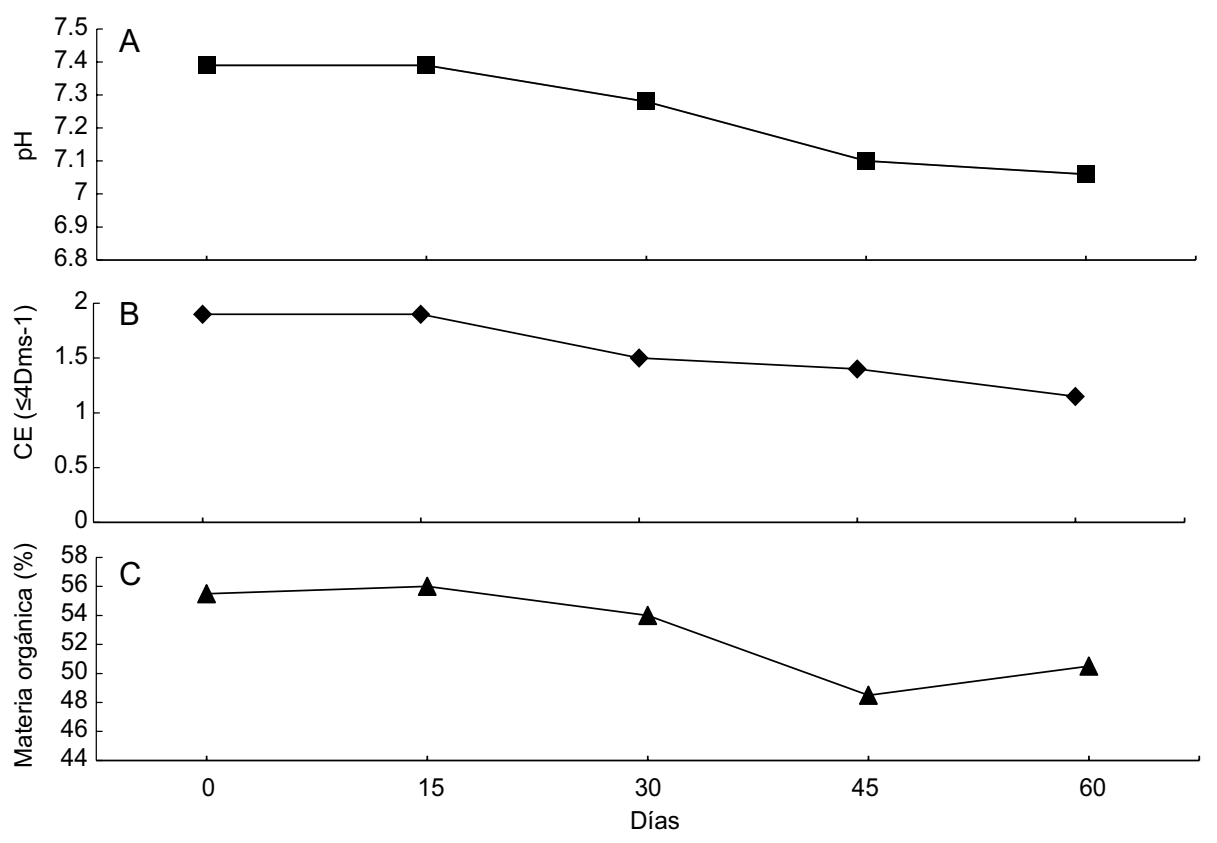

Fig. 2. Evaluación de (a) pH, (b) conductividad eléctrica (CE), y (c) materia orgánica durante el proceso de vermicompostaje 
rangos de 7 a 8 (David-Santoya et al. 2018, GonzálezSalas et al. 2018, Kováčik et al. 2018, Martínez et al. 2018), por lo que la vermicomposta elaborada a partir de lodo residual y estiércol equino es óptima, ya que promueve la disponibilidad de nutrientes y limita la de metales pesados.

La CE inició en $1.9 \mathrm{dS} / \mathrm{m}$ y al finalizar el proceso de vermicomposteo se obtuvo un valor de 1.15 dS/m (Fig. 2b). La CE mide la concentración de sales solubles presentes en el sustrato; esto significa que, a mayor CE, es mayor la concentración de sales (Havlin y Moebius-Clune 2012).

Para vermicompostas preparadas a base de residuos de podas de jardín y estiércol vacuno, Vázquez y Loli (2018) encontraron una CE de $7.16 \mathrm{dS} / \mathrm{m}$. Y en estudios realizados en vermicompostas elaborada con estiércol de conejo solo $\left(\mathrm{V}_{0}\right)$ y con la mezcla de lodo residual y este componente en proporción 10:90 $\left(\mathrm{V}_{1}\right), 30: 70\left(\mathrm{~V}_{2}\right)$, y 50:50 $\left(\mathrm{V}_{3}\right)$ se obtuvo una CE de $1.95,2.93,3.47$ y $4.17 \mathrm{dS} / \mathrm{m}$, respectivamente, variación proporcional a la concentración de lodo residual añadido (Soriano et al. 2008).

A diferencia de la vermicomposta de lodo residual y estiércol de conejo, la vermicomposta elaborada en esta investigación con las mismas proporciones (30:70), presentó una concentración de CE tres veces menor al utilizar estiércol equino en lugar del estiércol de conejo. Y para el caso de vermicompostas a base de podas de jardín y estiércol vacuno, en comparación con la vermicomposta de lodo residual y estiércol equino, la disminución de $\mathrm{CE}$ fue de 6.2 veces.

Havlin y Moebius-Clune (2012) recomiendan que la $\mathrm{CE}$ de un sustrato sea baja, en lo posible menor a $1 \mathrm{dS} / \mathrm{m}$, para facilitar el manejo de la fertilización y evitar problemas por fitotoxicidad en los cultivos. Y considerando los niveles admisibles de salinidad de la enmienda orgánica $(\leq 4 \mathrm{dS} / \mathrm{m}$ ) (Soriano et al. 2008), así como los parámetros establecidos en la NMX-FF-109-SCFI-2008 (SAGARPA 2008), la vermicomposta de lodos residuales y estiércol equino resulta de buena calidad y apta para su aplicación a cultivos.

El porcentaje de materia orgánica en la vermicomposta presentó fluctuaciones durante el proceso: disminuyó después de los 15 y hasta los 45 días de 56 a $48.5 \%$, y finalizó con $50.5 \%$ en el día 60 (Fig. 2c).

A diferencia de Zanor et al. (2018), que obtuvieron un porcentaje de materia orgánica de 59.61 para vermicomposta elaborada de lodo residual de afluentes de estiércol bovino; y en contraste con David-Santoya et al. (2018) que elaboraron una vermicomposta sólo con residuos orgánicos y obtuvieron un porcentaje muy bajo (8.4) de materia orgánica. La vermicomposta de lodo residual y estiércol equino de nuestro estudio, a pesar de presentar fluctuaciones durante el proceso, tuvo porcentajes de materia orgánica altos (50.5) que favorecerán el crecimiento y rendimiento de los cultivos.

La vermicomposta de lodo residual y estiércol equino, al igual que la elaborada por Morales-Munguía et al. (2009) con diferentes estiércoles, presenta fluctuaciones en el porcentaje de materia orgánica durante el proceso de estabilización y maduración; esto se debe, según Suthar y Singh (2008), a la fragmentación mecánica ejercida por la lombriz y a los cambios químicos y bioquímicos provocados por la digestión enzimática de este anélido, lo que da por resultado la producción de $\mathrm{CO}_{2}, \mathrm{H}_{2} \mathrm{O}$, iones minerales y MO estabilizada.

De acuerdo con algunos estudios, el tiempo necesario para generar una vermicomposta apta para la aplicación a cultivos requiere de 12 a 16 semanas (Campos-Medina et al. 2011); pero en estudios realizados por Zanor et al. (2018), se consideró una vermicomposta madura y apta para su uso a partir de las 10 semanas. De acuerdo con la Norma Oficial Mexicana NMX-FF-109-SCFI-2008 (SAGARPA 2008), la vermicomposta de estiércol apta para su manejo y aplicación debe cubrir el grado de estabilización orgánica en el producto terminado, mismo que puede verificarse por cualquiera de los siguientes parámetros: materia orgánica, relación $\mathrm{C} / \mathrm{N}$, humedad, $\mathrm{pH}$, conductividad eléctrica, capacidad de intercambio catiónico y densidad aparente.

A pesar de que la norma presenta parámetros exactos en su especificación físicas y químicas, no establece el tiempo necesario para el proceso de vermicompostaje. Sin embargo, se requiere disminuir los tiempos de elaboración, ya que como mencionan Acosta-Durán et al. (2013), uno de los factores importantes durante la elaboración de la vermicomposta es el tiempo, que implica gasto de insumos y, por ende, puede incrementar el costo de la misma.

La vermicomposta elaborada en esta investigación se estabilizó, cubriendo los parámetros establecidos por la norma NMX-FF-109-SCFI-2008, y fue aplicada a cultivos de albahaca a las 8.5 semanas de su elaboración.

\section{Evaluación de la productividad}

Todos los tratamientos con vermicomposta en las plantas de albahaca incrementaron significativamente la tasa absoluta de crecimiento (TAC), medida en peso, en una relación dosis-respuesta (Cuadro III). Se incrementó el peso un 25, 45 y $66 \%$ 
en los tratamientos $\mathrm{T}_{20}, \mathrm{~T}_{40}$ y $\mathrm{T}_{60}$, respectivamente, con relación al testigo relativo $\mathrm{CT}_{0}$. Con relación al testigo absoluto se obtuvieron incrementos de 1.9, $2.7,3.3$ y 3.9 veces en los tratamientos $\mathrm{CT}_{0}, \mathrm{~T}_{20}, \mathrm{~T}_{40}$ y $_{60}$, respectivamente. Estos resultados indican que posiblemente el suelo empleado en este trabajo, que provenía de cultivos de una zona productora de papa cultivada durante seis años y que fue utilizado en el $\mathrm{CT}_{0}$, contenía nutrientes residuales de la fertilización constante.

CUADRO III. TASAABSOLUTA DE CRECIMIENTO (TAC) EVALUADA EN LA ALTURA Y EL PESO EN PLANTAS DE ALBAHACA (Ocimum basilicum L.)*

\begin{tabular}{lcc}
\hline \multirow{2}{*}{ Tratamiento } & \multicolumn{2}{c}{ Tasa absoluta de crecimiento TAC } \\
\cline { 2 - 3 } & Longitud (cm/día)** & Peso $(\mathrm{mg} /$ día)** \\
\hline $\mathrm{C}$ & $0.460 \pm 0.07^{\mathrm{A}}$ & $24 \pm 0.01^{\mathrm{A}}$ \\
$\mathrm{CT}_{0}$ & $0.821 \pm 0.08^{\mathrm{B}}$ & $71 \pm 0.006^{\mathrm{B}}$ \\
$\mathrm{T}_{20}$ & $0.782 \pm 0.10^{\mathrm{AB}}$ & $89 \pm 0.009^{\mathrm{BC}}$ \\
$\mathrm{T}_{40}$ & $0.756 \pm 0.10^{\mathrm{AB}}$ & $103 \pm 0.008^{\mathrm{C}}$ \\
$\mathrm{T}_{60}$ & $1.015 \pm 0.08^{\mathrm{B}}$ & $118 \pm 0.008^{\mathrm{C}}$ \\
\hline
\end{tabular}

*Fuente: Hunt (1982)

**Promedio \pm error estándar. Letras diferentes denotan diferencias significativas de acuerdo con la prueba de Tukey a una $\mathrm{p} \leq 0.05$

En contraste, la longitud permaneció constante en todos los tratamientos y no tuvo diferencias significativas con relación el testigo relativo $\left(\mathrm{CT}_{0}\right)$. La longitud se incrementó en todos los tratamientos incluido el $\mathrm{CT}_{0}$ respecto a $\mathrm{C}$ (absoluto), lo que reafirma la posibilidad de que el suelo de $\mathrm{CT}_{0}$ tuviera trazas o residuos de nutrientes.

Estos resultados indican que el cultivo presentó un incremento de biomasa al aplicarse la vermicomposta. Inclusive, esta respuesta fue observada en un experimento realizado en invierno en las mismas condiciones (datos no mostrados).

Se ha demostrado que los efectos de vermicompostas de diferentes composiciones y condiciones de elaboración incrementan el crecimiento, desarrollo y la productividad de una amplia gama de cultivos. Sin embargo, hay pocos estudios referentes al efecto de vermicompostas elaboradas con lodos residuales sobre el efecto agronómico de las plantas, así como en la bioquímica de estas.

Se ha estudiado el efecto de la aplicación directa de lodos residuales en frijoles (Negrín y Jiménez 2012) y rábano (Silva-Leal et al. 2013), obteniéndose un incremento significativo en variables agronómicas como longitud, diámetro de tallo, número de hojas y biomasa total; pero la utilización de lodos no supera las 9 t/ha y su aplicación presenta un riesgo elevado para la salud humana.

Para los productos del vermicompostaje, por ejemplo, en ensayos con plántulas de Solanum lycopersicum, el uso de humatos de vermicompostaje incrementó de manera significativa la longitud y el peso fresco en 61 y $56 \%$, respectivamente, con relación al tratamiento control (Chiquito-Contreras et al. 2018). Por otro lado, la vermicomposta generada a partir de residuos vegetales, cascarilla de cacao, y estiércol de ovino, estimuló el crecimiento del chile habanero (Capsicum chinense Jacquin) e incrementó el porcentaje de germinación. La combinación de vermicomposta y suelo (1:1) incrementó de forma significativa la altura y biomasa fresca de las plántulas de chile habanero al compararse con el testigo (David-Santoya et al. 2018).

Roblero-Ramírez et al. (2014) mencionan que el efecto de la vermicomposta sobre el desarrollo del tomate influyó de manera importante en el rendimiento de las plantas, incrementando el número de frutos y su peso 1.2 y 14.2 veces respecto al testigo.

En correspondencia con lo anterior, DavidSantoya et al. (2018) señalaron que la respuesta de crecimiento y rendimiento de las plantas está influenciada por el uso de diferentes sustratos para la elaboración de vermicompostas, lo cual demuestra de manera consistente que los residuos orgánicos vermicomposteados tienen efectos benéficos sobre el crecimiento de la planta, independientemente del origen de los sustratos y las transformaciones y disponibilidad de los elementos nutritivos.

De acuerdo con Guo et al. (2015), la vermicomposta contiene algunos reguladores del crecimiento, lo que estimula en las plantas de maíz la asimilación de nutrientes para generar una mayor biomasa y rendimiento. Por lo anterior, la vermicomposta elaborada con lodo residual y estiércol equino incrementó significativamente la productividad de las plantas de albahaca. Para saber si el uso de esta vermicomposta afecta al cultivo por el uso de lodo residual, se evaluó la actividad antioxidante de las plantas de albahaca a través del contenido de compuestos fenólicos y la capacidad antioxidante evaluada mediante la técnica DPPH.

\section{Cuantificación del contenido de compuestos fe- nólicos totales}

Todos los tratamientos con vermicomposta y el $\mathrm{CT}_{0}$ tuvieron estadísticamente menor contenido de fenoles respecto al control absoluto C (Fig. 3). 


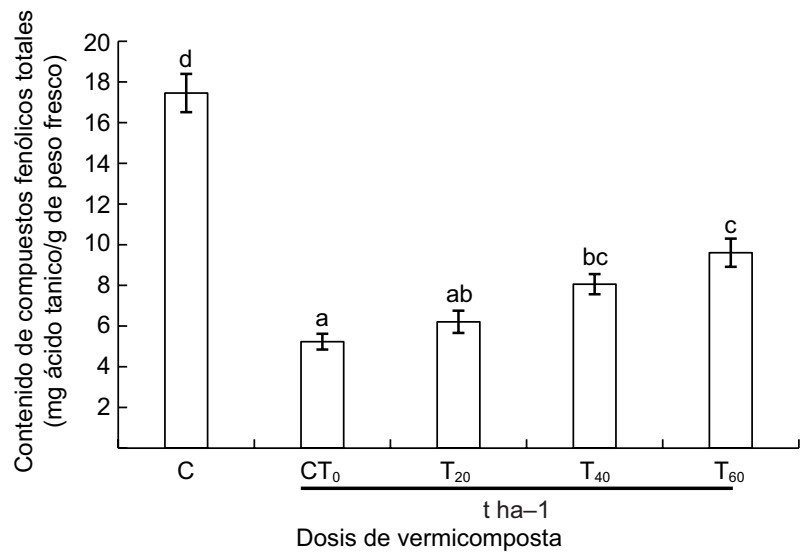

Fig. 3. Contenido de compuestos fenólicos totales de plantas de albahaca (Ocimum basilicum L.) cultivadas en vermicomposta elaborada con lodo residual y estiércol equino. Letras diferentes denotan diferencias significativas de acuerdo con la prueba de Tukey a una $\mathrm{p} \leq 0.05$

De la Rosa et al. (2009) señalan que el estrés induce la síntesis y acumulación de compuestos fenólicos y, dado su carácter antioxidante, éstos desempeñan un papel protector celular clave en circunstancias de estrés.

En estudios realizados en tomates, Toor et al. (2006) evaluaron el efecto de la nutrición en la actividad antioxidante, demostrando que la fuente de nutrientes desempeña una función importante en la determinación de los componentes antioxidantes; por este motivo, los resultados de la aplicación de vermicomposta de lodo residual y estiércol equino pueden atribuirse a la disponibilidad de macronutrientes. En este sentido, Nguyen y Niemeyer (2008) confirmaron que la acumulación fenólica está relacionada con la disponibilidad de nutrientes como nitrógeno, fósforo, potasio y calcio; y de acuerdo con Salas et al. (2018), la falta de nutrición pudo haber estimulado las rutas enzimáticas de formación de compuestos antioxidantes y por ende la acumulación de compuestos fenólicos encontrados en el tratamiento control absoluto.

Por lo anterior es posible señalar que la adición de vermicomposta como fertilizante no ocasiona estrés en comparación con el control absoluto.

\section{Cuantificación de la capacidad antioxidante}

La capacidad antioxidante evaluada mediante el método DPPH para albahaca (O. basilicum L.) solo se incrementó significativamente en el tratamiento $\mathrm{T}_{60}$, con relación a los demás tratamientos y controles (Fig. 4).

Fortis-Hernández et al. (2018) explican que, para el cultivo de tomate, los fertilizantes orgánicos basados en vermicompostas inducen la concentración de

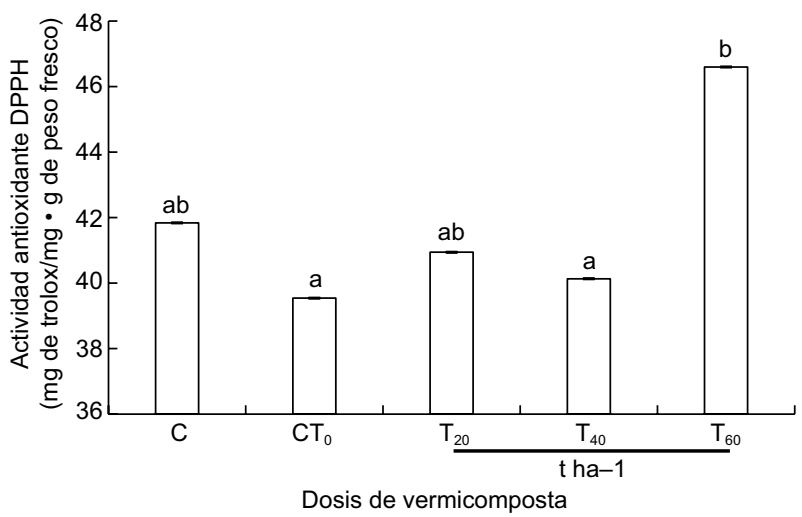

Fig. 4. Actividad antioxidante de 2,2-difenil-1-picrilhidracilo (DPPH) de plantas de albahaca (Ocimum basilicum L.) cultivadas en vermicomposta elaborada con lodo residual y estiércol equino. Letras diferentes denotan diferencias significativas de acuerdo con la prueba de Tukey a una $\mathrm{p} \leq 0.05$

licopeno en frutas, así como mejoras en el contenido de fenoles y antioxidantes.

De acuerdo con López-Martínez et al. (2017), la capacidad antioxidante y el rendimiento de las plantas se ven afectados por la concentración de nutrición aplicada; aunado a esto, Omar et al. (2012) mencionan que la fertilización tiene influencia en la calidad fitonutricional de los cultivos, y en comparación con la fertilización de inorgánicos, se comprobó que los fertilizantes orgánicos aumentan el contenido de antioxidantes en las plantas.

Por lo anterior, la capacidad antioxidante encontrada en los tratamientos $\mathrm{T}_{60}$ se puede atribuir a la nutrición proporcionada por la vermicomposta de lodo residual y estiércol equino.

\section{CONCLUSIONES}

La elaboración de vermicomposta de lodo residual y estiércol equino cubrió los parámetros establecidos por la NOM-021-RECNAT-2000 en 80 días, y su uso incrementó la tasa absoluta de crecimiento sin alterar la actividad antioxidante y el contenido de compuestos fenólicos en las plantas de albahaca (Ocimum basilicum L.).

\section{REFERENCIAS}

Abe N., Murata T. y Hirota A. (1998). Novel DPPH radical scavengers, bisorbicillinol and demethyltrichodimerol, from a fungus. Biosci. Biotechnol. Biochem. 62 (4), 661-666. DOI: 10.1271/bbb.62.661 
Acosta-Durán C.M., Solís-Pérez O., Villegas-Torres O.G. y Cardoso-Vigueros L. (2013). Precomposteo de residuos orgánicos y su efecto en la dinámica poblacional de Eisenia foetida. Agron. Costarricense 37 (1), 127-139.

Alloway B.J. (2013). Sources of heavy metals and metalloids in soils. En: Heavy metals in soils (B. Alloway, Ed.). Springer, Dordrecht, Holanda, 11-50.

DOI: $10.1007 / 978-94-007-4470-7 \_2$

Arancon N.Q. y Edwards C.A. (2011). The use of vermicomposts as soil amendments for production of field crops. En: Vermiculture technology: Earthworms, organic wastes, and environmental management (Edwards C.A., Arancon N.Q. y Sherman R.L., Eds.). CRC Press, Boca Raton, EUA, 129-151.

DOI: $10.1201 / \mathrm{b} 10453-11$

BID (2013). IDB-TN-521. Tratamiento de aguas residuales en México. Nota técnica. Banco Interamericano de desarrollo. Sector de Infraestructura y Medio Ambiente, México, 42 pp. DOI: 10.18235/0000140

Bloem J., Schouten A.J., Sørensen S.J., Rutgers M., van der Werf A.K. y Breure A.M. (2006). Monitoring and evaluating soil quality. En: Microbiological methods for assessing soil quality (Bloem J., Hopkins D.W y Benedetti A., Eds.). CABI, Reino Unido, 23-49. DOI: 10.1079/9780851990989.0023

Cabanillas C., Stobbia D. y Ledesma A. (2013). Production and income of basil in and out of season with vermicomposts from rabbit manure and bovine ruminal contents alternatives to urea. J. Clean Prod. 47, 77-84. DOI: 10.1016/j.jclepro.2013.02.012

Campos-Medina E., Velázquez-Rodríguez A. y GómezHinojos A.M. (2011). Predicción y comparación de transferencia de nutrientes de dos tipos de vermicomposteo de lodos residuales a suelos forestales. Quivera $13(1), 1-15$.

Carbonell G., Torrijos M., Rodríguez J. A. y Ángel Porcel M. (2016). Uptake and metal transfer from biosolidamended soil to tomato (Solanum lycopersicum Mill L.) plants. J. Plant Chem. Ecophysiol. 1 (1), 1002.

Charles D.J. (2012). Antioxidant properties of spices, herbs and other sources. Springer, Norway, EUA, 610 pp. DOI: $10.1007 / 978-1-4614-4310-0$

Chiquito-Contreras R.G., Reyes-Pérez J.J., Troyo-Diéguez E., Rueda-Puente E.O., Torres-Rodríguez J.A. y Murillo-Amador B. (2018). Crecimiento de plántulas de tomate (Solanum lycopersicum L.) tratadas con humato de vermicompost. Rev. Mex. Cienc. Agríc. 9 (20), 4189-4197. DOI: 10.29312/remexca.v0i20.989

Cuypers A., Remans T., Weyens N., Colpaert J., Vassilev A. y Vangronsveld J. (2013). Soil-plant relationships of heavy metals and metalloids. En: Heavy metals in soils (Alloway B., Ed.). Springer, Dordrecht, Holanda, pp 161-193. DOI: 10.1007/978-94-007-4470-7_6
David-Santoya J.J.E., Gómez-Álvarez R., Jarquín-Sánchez A. y Villanueva-López G. (2018). Caracterización de vermicompostas y su efecto en la germinación y crecimiento de Capsicum chinense Jacquin. Ecosist. Recur. Agropec. 5 (14), 181-190.

DOI: 10.19136/era.a5n14.1465

De la Rosa L.A., Álvarez-Parrilla E. y González-Aguilar G.A. (2009). Fruit and vegetable phytochemicals: chemistry, nutritional value and stability. Wiley Blackwell. Nueva Delhi, India, 367 pp.

DOI: 10.1002/9780813809397

Fortis-Hernández M., Preciado-Rangel P., Segura-Castruita M.A., Mendoza-Tacuba L., Gallegos-Robles M.A., Hernández J.L.G. y Vásquez-Vásquez C. (2018). Changes in nutraceutical quality of tomato under different organic substrates. Hortic. Bras. 36 (2), 189-194.

DOI: 10.1590/s0102-053620180207

Garg V.K., Chand S. Chhillard A. y Yadav A. (2005). Growth and reproduction of Eisenia foetida in various animal wastes during vermicomposting. Appl. Ecol. Environ. Res. 2, 51-59.

DOI: $10.15666 /$ aeer/0302_051059

González-Salas U., Gallegos-Robles M.Á., VázquezVázquez C., García-Hernández J.L., Fortis-Hernández M. y Mendoza-Retana S.S. (2018). Productividad de genotipos de maíz forrajero bajo fertilización orgánica y propiedades físico-químicas del suelo. Rev. Mex. Cienc. Agríc. 20, 4331-4341.

DOI: $10.29312 /$ remexca.v0i20.1002

Guo L., Wu G., Li C., Liu W., Yu X., Cheng D. y Jiang G. (2015). Vermicomposting with maize increases agricultural benefits by $304 \%$. Agron. Sustain. Dev. $35,1149-1155$.

DOI: $10.1007 / \mathrm{s} 13593-015-0307-0$

Havlin J. y Moebius-Clune B. (2012). Chemical Properties of soil: Soil fertility and Nutrient Management. In: Know soil, know life (D.L. Lindbo, D.A. Kozlowski y C. Robinson, Eds.). Soil Sci. Soc. Am. EUA. pp. 69-81.

DOI: $10.2136 / 2012 . k n o w s o i l . c 4$

Holguín-Calderón E., Morales-Rodríguez M., Vicenciode la Rosa M. y Morales-de Casas M. (2014). Lodos residuales: métodos de tratamiento, estabilización y aprovechamiento. Vidsupra 6 (2), 61-66.

Hunt R. (1982). Plant growth curves. The functional approach to plant growth analysis. 1a ed. Edward Arnold, Londres, Reino Unido, 1982 pp.

ICAMEX (2008). CE:2017/03/06/13. Manual para el establecimiento y manejo de instalaciones lombrícolas. Manual. Instituto de Investigación y Capacitación Agropecuaria, Acuícola y Forestal del Estado de México, Estado de México, México, 27 pp. 
INIFAP (2004). Mayor producción de alfalfa fertilizando el suelo con biosólidos. Folleto Técnico No. 18. Instituto Nacional de Investigaciones Forestales, Agrícolas y Pecuarias, Fundación Produce Chihuahua, Chihuahua, Chihuahua, México, 51 pp.

INEGI (2007). E 14-2. Conjunto de datos vectorial edafológico, serie II, escala 1:250 000 (continuo nacional). Instituto Nacional de Estadística y Geografía, Ciudad de México, México.

IUSS (2007). Base referencial mundial del recurso suelo. Primera actualización. Informes sobre Recursos Mundiales de Suelos No. 103. IUSS Grupo de Trabajo WRB, FAO, Roma, Italia, 117 pp.

Kováčik P., Šalamun P., Smoleń S. y Renčo M. (2018). Impact of vermicompost as component of growing medium on phytomass formation of radish (Raphanus sativus L.). Agriculture 64 (3), 106-115.

DOI: 10.2478/agri-2018-0011

Lim S.L., Lee L.H. y Wu T.Y. (2016). Sustainability of using composting and vermicomposting technologies for organic solid waste biotransformation: Recent overview, greenhouse gases emissions and economic analysis. J. Clean. Prod. 111, 262-278.

DOI: 10.1016/j.jclepro.2015.08.083

López-Martínez J., Salas-Pérez L., Valenzuela-Soto R., Borroel-García V., Preciado-Rangel P. y RamírezSeañez A. (2017). Efecto del potasio en el contenido fenólico y capacidad antioxidante de Ocimum basilicum L. Rev. Mex. Cienc. Agríc. 8 (1), 133-145.

DOI: $10.29312 /$ remexca.v8i1.77

Lugo J., del Águila P., Vaca R., Casas-Hinojosa I. y Yáñez-Ocampo G. (2017). Abono orgánico elaborado con lodo residual y estiércol equino a través de vermicomposteo: una propuesta como mejorador de suelos. Rev. Int. Contam. Ambie. 33 (3), 475-484.

DOI: $10.20937 /$ rica.2017.33.03.10

Martínez L.E., Vallone R.C., Piccoli P.N. y Ratto S.E. (2018). Evaluación de propiedades edáficas químicas, biológicas, rendimiento y composición vegetal en un viñedo de Mendoza (Argentina) con diferentes tipos y modos de aplicación de abono orgánico. Universidad Nacional de Cuyo. Revista de la Facultad de Ciencias Agrícolas 50 (1), 17-32.

Morales-Munguía J.C., Fernández-Ramírez M.V., Montiel-Cota A. y Peralta-Beltrán B.C. (2009). Evaluación de sustratos orgánicos en la producción de lombricomposta y el desarrollo de lombriz (Eisenia foetida). Biotecnia 11 (1), 19-26. DOI: 10.18633/bt.v11i1.49

Negrín B.A. y Jiménez P.Y. (2012). Evaluación del efecto agronómico del biosólido procedente de una planta de tratamiento por digestión anaerobia de residuales pecuarios en el cultivo del frijol (Phaseolus vulgaris L). Cul. Trop. 33 (2), 13-19.
Nguyen P.M. y Niemeyer E.D. (2008). Effects of nitrogen fertilization on the phenolic composition and antioxidant properties of basil (Ocimum basilicum L.). J. Agric. Food Chem. 56 (18), 8685-8691.

DOI: $10.1021 / \mathrm{jf} 801485 \mathrm{u}$

Omar N.F., Hassan S.A., Yusoff U.K., Abdullah N.A.P., Wahab P.E.M. y Sinniah U.R. (2012). Phenolics, flavonoids, antioxidant activity and cyanogenic glycosides of organic and mineral-base fertilized cassava tubers. Molecules 17 (3), 2378-2387.

DOI: $10.3390 /$ molecules 17032378

Roblero-Ramírez H.R., Nava-Pérez E., Valenzuela-Quiñónez W., Báez C., Ricardo J. y Rodríguez-Quiroz G. (2014). Evaluación de cinco dosis de vermicomposta en el cultivo de tomate (Solanum lycopersicum) en Sinaloa, México. Rev. Mex. Cienc. Agríc. 8, $1495-$ 1500 .

DOI: 10.29312/remexca.v0i8.1108

Rodríguez J.J.H., Mosqueda M.C.R. y Elizarraraz R.A. (2018). Análisis de biosólidos para su uso agrícola en una hortaliza. Jóvenes en la Ciencia 3, 340-344.

Romero R.C.O., Ocampo M.J., Sandoval C.E. y Tobar R.J.R. (2018). Evaluación de sustratos para la producción de lombriz de tierra (Eisenia foetida). Ctro. Agr. 45 (4), 68-74.

SAGARPA (2008). Norma Mexicana NMX-FF-109-SCFI-2008. Humus de lombriz (lombricomposta) especificaciones y métodos de prueba. Secretaria de Comercio y Fomento Industrial, Secretaría de Agricultura, Ganadería, Desarrollo Rural, Pesca y Alimentación. Diario Oficial de la Federación, 26 de mayo.

Salas P.L., Borroel-García V.J., Ramírez-Aragón M.G. y Moncayo L.M.R. (2018). Efecto de la adición de ácido ascórbico y té de composta en la producción y capacidad antioxidante de forraje hidropónico de maíz. Nova Scientia 10 (20), 47-63.

DOI: $10.21640 /$ ns.v10i20.1168

SEMARNAT (2002). Norma Oficial Mexicana NOM021-RECNAT-2000. Fertilidad, salinidad y clasificación de suelos. Estudios, muestreo y análisis. Secretaría de Medio Ambiente y Recursos Naturales. Diario Oficial de la Federación, 31 de diciembre.

Silva-Leal J.A., Bedoya D.F. y Torres-Lozada P. (2013). Evaluation of potential application disinfected of biosolids on radish culture. Cienc. Suelo 6 (2), 155-164.

Singh S., Singh J. y Vig A.P. (2016). Earthworm as ecological engineers to change the physico-chemical properties of soil: Soil vs vermicast. Ecol. Eng. 90, 1-5. DOI: $10.1016 /$ j.ecoleng.2016.01.072

Soriano M.D., Molina M., Linares J., Pons J. y Ingelmo F. (2008). Estabilización de residuos de vinazas y lodos de depuradora tras un proceso de vermicomponstaje con estiércol de conejo en condiciones de laboratorio. 
Memorias. Simposio Iberoamericano de Ingeniería de Residuos. Castellón, España. 23 y 24 de junio. CD-ROM.

Suthar S. y Singh S. (2008). Vermicomposting of domestic waste by using two epigeic earthworms (Perionyxexcavatus and Perionyxsansibaricus). J. Environ. Sci. Technol. 5 (1), 99-106.

DOI: $10.1007 / \mathrm{BF} 03326002$

Toor R.K., Savage G.P. y Heeb A. (2006). Influence of different types of fertilizers on the major antioxidant components of tomatoes. J. Food Compos. Anal. 19 (1), 20-27.

DOI: $10.1016 /$ j.jfca.2005.03.003

Utria-Borges E., Cabrera-Rodríguez J.A., ReynaldoEscobar I.M., Morales-Guevara, D., Fernández A.M. y Toledo T.E. (2008). Utilización agraria de los biosólidos y su influencia en el crecimiento de plántulas de tomate (Lycopersicon esculentum Mill). Rev. Chapingo Ser. Hortic.14 (1), 33-39.

DOI: $10.5154 /$ r.rchsh.2006.02.009
Vázquez J. y Loli O. (2018). Compost y vermicompost como enmiendas en la recuperación de un suelo degradado por el manejo de Gypsophila paniculata. Sci. Agropecu. 9 (1), 43-52.

DOI: 10.17268/sci.agropecu.2018.01.05

Vicencio-de la Rosa M., Pérez-López M., Medina-Herrera E. y Martínez-Prado M.A. (2011). Producción de composta y vericomposta a partir de los lodos de la planta de tratamiento de aguas residuales de un rastro. Rev. Int. Contam. Ambie. 27 (3), 263-270.

Waterman P.G. y Mole S. (1994). Analysis of phenolic plant metabolites. Blackwell Scientific Publication, Oxford, Reino Unido, 238 pp.

Zanor G.A., López-Pérez M.E., Martínez-Yáñez R., Ramírez-Santoyo L.F., Gutiérrez-Vargas S. y LeónGalván M.F. (2018). Mejoramiento de las propiedades físicas y químicas de un suelo agrícola mezclado con lombricompostas de dos efluentes de biodigestor. Ing. Investig. Tecnol. 19 (4), 1-10.

DOI: $10.22201 /$ fi.25940732e.2018.19n4.036 